\title{
Congenital Agenesis of the Gallbladder with the Hypogenetic Cystic Duct Definitely Diagnosed by Peroral Cholangioscopy
}

\author{
Kenji Sasaki*
}

Department of Internal Medicine, Shiogama City Hospital, Kouzumachi, Shiogama, Miyagi, 985-0054, Japan

Received: October 03, 2014; Accepted: November 14, 2014; Published: November 28, 2014

*Corresponding author: Kenji Sasaki, Department of Internal Medicine, Shiogama City Hospital, 7-1, Kouzumachi, Shiogama, Miyagi, 985-0054, Japan, Tel: +81-22-364-5521; Fax: +81-22-364-5529; E-mail: kydosarnymai@aria.ocn.ne.jp

\begin{abstract}
A 59-year-old Japanese female presented with a fewday history of abdominal pain, nausea, vomiting, icterus, and low-grade fever. Her past and family histories were non-contributory. Laboratory data showed elevated serum hepatic and pancreatic enzymes, hyperbilirubinemia, and leukocytosis. Computed Tomography (CT) demonstrated the dilated biliary tree obstructed by a stone in the distal part and the swollen pancreas with the enhanced peripancreatic adipose tissue. No entopic or ectopic gallbladder was detected by Ultrasonography (US), CT, or Magnetic Resonance Cholangiopancreatography (MRCP). The papilla of Vater was patent-orificed. Though failed to visualize the gallbladder, Endoscopic Retrograde Cholangiography (ERC) showed a stone in the distal and a short, thin, blind tube arising from the mid extrahepatic biliary tree. She underwent Endoscopic Sphincterotomy (EST) and choledocholithotomy under the preoperative diagnosis of Congenital Agenesis of the Gallbladder (CAGB) with the hypogenetic cystic duct complicated with choledocholithiasis triggering acute cholangitis and pancreatitis. Peroral cholangioscopy revealed the tube to be a crescent-folded cul-de-sac running parallel with and draining into the common hepatic duct, the vestige of the abortive primordium which should have developed into the full-fledged cystic duct and gallbladder, confirming the diagnosis. She has kept an uneventful post-operative course. This is the fifth case of CAGB with the hypogenetic cystic duct and the first in the world, in which the diagnosis was established by peroral findings. Emphasized are the crucial role of peroral cholangioscopy and ERC and the importance of awareness of the anomaly to obviate unnecessary, potentially dangerous exploration through the non-natural orifices in quest of the phantom gallbladder.
\end{abstract}

Keywords: Agenesis of the gallbladder; Hypogenesis of the cystic duct; Peroral cholangioscopy; ERCP

\section{Introduction}

CAGB is a rare congenital anomaly [1], which is, in adults, serendipitously detected at autopsy or exploratory laparotomy in most cases. Though per se usually poses no problems, it sometimes complicates adult cases exhibiting biliary symptoms, defying diagnosis [2-5]. It was once stated that the preoperative diagnosis of CAGB was extremely difficult or even impossible [25] and it still remains exceedingly hard to correctly diagnose the anomaly before an operation despite high accuracy of modern diagnostic techniques [6-8]. Though it is usually associated with the absence of the cystic duct, there have been four cases of the anomaly in the world, so far, reported to be accompanied by the hypogenetic cystic duct $[6,7,9,10]$, whose presence renders it even more difficult to correctly diagnose the anomaly.

This report presents a case of such an anomaly successfully treated with EST and choledocholithotomy under the preoperative diagnosis of CAGB with the hypogenetic cystic duct complicated with choledocholithiasis inducing acute cholangitis and pancreatitis, which was definitely confirmed by peroral cholangioscopy without resorting to exploration through the non-natural orifices.

\section{Case Report}

A 59-year-old Japanese spinster presented with a few-day history of abdominal pain, nausea, vomiting of food residue and/ or gastric juice, mild icterus, and low-grade fever. She suffered from miliary tuberculosis complicated with meningitis at age zero, which rendered her right hemiparetic. She went deaf due to streptomycin administered then. She suffered from dysentery at age three. Her other past and family histories were noncontributory. The abdomen was protruding and soft and there was no organomegaly. She had pain on pressure around the umbilicus.

Abdominal X-ray showed no niveaux or free air. Only the dilated extrahepatic bile duct was barely depicted through her extremely thick adipose tissue by US. CT showed a round, solitary, calcified-surfaced stone in the intrapancreatic bile duct with the dilated upstream and increased density in the adipose tissue around the swollen pancreatic head (Figure 1). The main pancreatic duct was not dilated and there was no peripancreatic fluid. No entopic or ectopic gallbladder was detectable by US, CT, or MRCP. 
Laboratory data showed conjugated type-dominant hyperbilirubinemia (total bilirubin $3.6 \mathrm{mg} / \mathrm{dl}$, normal $0.2-1.1$, direct bilirubin $2.5 \mathrm{mg} / \mathrm{dl}$, normal 0.0-0.4), moderate liver dysfunction (AST 677, normal 10-38, ALT 555, normal 5-40, Al-P 1,025, normal 104-338, $\boldsymbol{\gamma}$-GTP 561, normal 8-45, LDH $714 \mathrm{IU} / \mathrm{l}$, normal 100-230), moderately elevated serum amylase (506 IU/l, normal 40-130), lipase (370 IU/l, normal 13-49), and CRP (4.9 $\mathrm{mg} / \mathrm{dl}$, normal 0.0-0.3), and moderate leukocytosis (WBC 15,400, normal 3,500-9,500).

Esophagogastroduodenoscopy showed the patent-orificed papilla of Vater. ERC demonstrated a stone measuring $10.0 \times 18.6$ $\mathrm{mm}$ in diameter in the dilated, distal bile duct measuring $15.7 \mathrm{~mm}$ in maximum diameter and a thin, short, rudimentary, blind duct measuring $4.0 \mathrm{~mm}$ in width and $17.0 \mathrm{~mm}$ in length originating from the mid extrahepatic bile duct and running cephalad parallel to it (Figure 2). No entopic or intrahepatic gallbladder was detected (Figure 3). Lack of previous history suggestive of biliary diseases and too short a duration of the present symptoms excluded the possibility of the scleroatrophic gallbladder. The gallbladder in the aberrant positions was also ruled out by the above mentioned examinations. Under the preoperative diagnosis of CAGB with the hypogenetic cystic duct complicated with choledocholithiasis inducing acute cholangititis and pancreatitis, EST was performed and a stone was successfully retrieved (Figure 4), which resulted in immediate improvement of her general status and laboratory data. Subsequent peroral cholangioscopy clearly showed the duct to be a crescent-folded cul-de-sac arising from and running cephalad parallel with the common hepatic duct (Figures 5a-5c), undoubtedly revealing it to be the hypogenetic cystic duct, the vestige of the abortive primordium which should have developed into the full-fledged cystic duct and gallbladder. No other abnormalities were detected in the papillary orifice through the bifurcation of the hepatic ducts. Thus the preoperative diagnosis was definitely proven accurate on the basis of the endoscopic characteristics satisfying Frey et al.'s two diagnostic criteria [2] She has kept an uneventful post-operative course for two years.

\section{Discussion}

CAGB is a rare anomaly with a reported incidence of 1:7,500 in

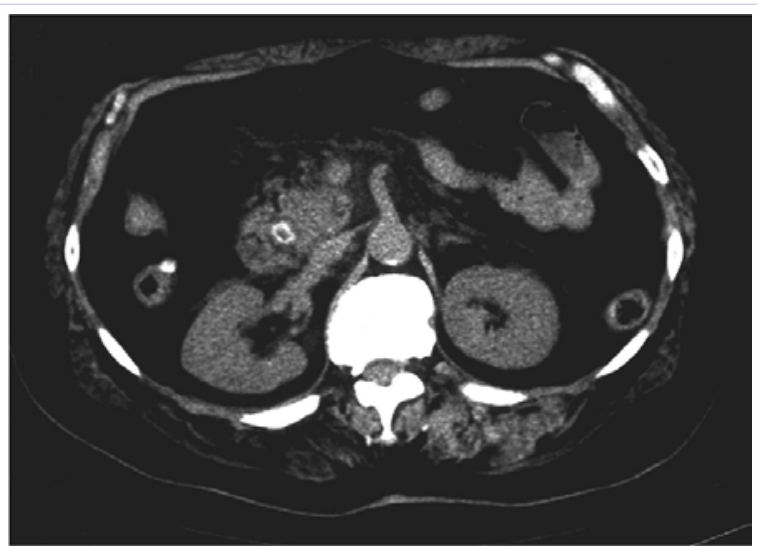

Figure 1: Plain CT showing a solitary stone with surface calcification in the intrapancreatic bile duct.

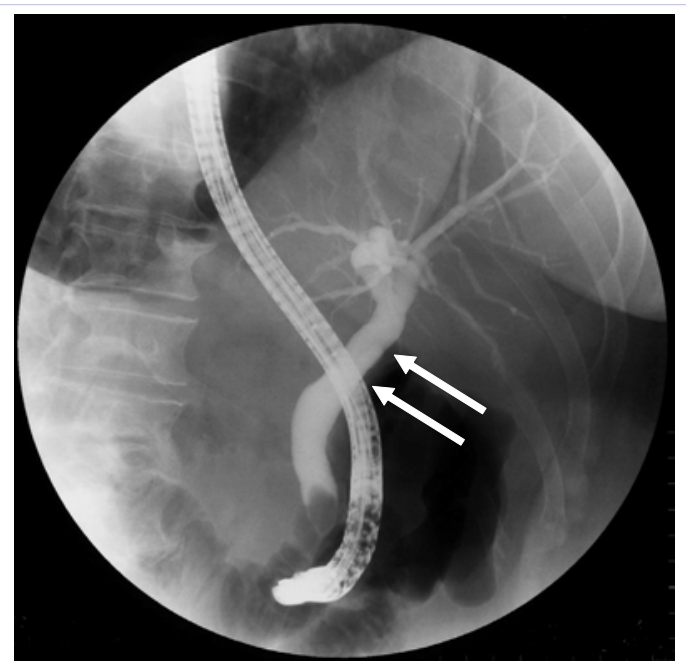

Figure 2: ERC taken in the semi-prone position. A short, thin, rudimentary cystic duct $(\uparrow \uparrow)$ is demonstrated originating from and running cephalad parallel to the mid extrahepatic bile duct. A gallstone is seen in the distal end.

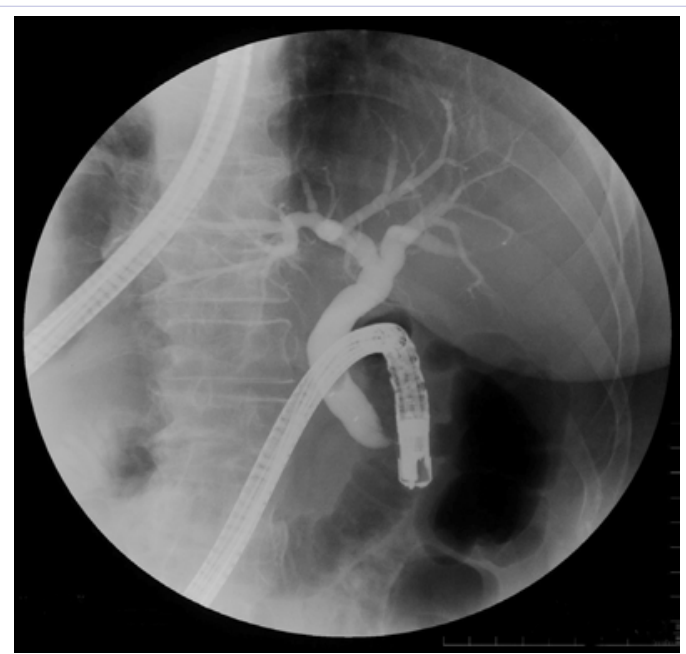

Figure 3: ERC taken in the almost prone position showing no entopic or intrahepatic gallbladder.

autopsy cases [1]. But, since more than half of the cases reported to be the anomaly, so far, did not satisfy the strict diagnostic criteria laid down by Frey et al. [2], the total number of CAGB still needs to be reevaluated [3]. The anomaly was already known in the ancient Greece: Aristotle dealt with it from the teleological point of view [11]. It is interpreted as the anomaly attributed to the disturbed development of the omphaloenteric and umbilical veins located on each side of the primordium of the gallbladder during the third week of gestation, which results in the lack of the anlage [12].

The definite proof of CAGB necessitates elimination of the conditions which could be mistaken for the anomaly, such as the ectopic or scleroatrophic gallbladder [2]. Cases of the gallbladder located in the aberrant positions have been described, such as 
in the retroperitoneum [13], suprahepatic space [14], falciform ligament [15], liver [16] and anterior abdominal wall [17]. Some of them were detected by CT $[13,14]$ and the others are considered clearly detectable by it [15-17]. No structures reminiscent of the gallbladder were revealed at such locations by any diagnostic modalities, so that the possibility of the ectopic gallbladder is excluded in this case.

The present case, unlike Frey et al.'s case four [2], had no previous history suggestive of hepatobiliary diseases and complained of no severe biliary symptoms only for too short a period to suffer from necrosis of the gallbladder. It is also inconceivable, even if an intense inflammatory reaction had occurred in the region of the gallbladder, that the organ and the major part of the cystic duct proximal to it went scleroatrophic, leaving the rest of the duct patent and that the cholecystolith migrated to the choledochus to become a large stone to trigger acute cholangitis and pancreatitis for the first time after a long dormant interval. It is more reasonable to consider the stone to have been formed in the choledochus de novo rather than in the illusory gallbladder. Sufficient ground is, therefore, lacking to suspect the present case to be the scleroatrophic gallbladder.

Frey et al. [2] proposed the two diagnostic criteria to prove the cases of CAGB: the operative findings confirmed by cholangiography and visualization of the biliary tract by the operative dissection from the duodenum to the bifurcation of the hepatic ducts [2]. Based on the criteria, some authors stressed the need of thorough surgical exploration [4], exploratory laparotomy with intraoperative cholangiography [18], or laparoscopy combined US [6]. In the present case, CT and MRCP failed to depict the entopic or ectopic gallbladder and ERC demonstrated no entopic or intrahepatic gallbladder but the dilated extrahepatic bile duct with an incompletely developed blind duct in the mid bile duct, which appeared to be the rudimentary cystic duct. These findings and awareness of the anomaly entity led to the preoperative diagnosis of CAGB with the hypogenetic cystic duct.

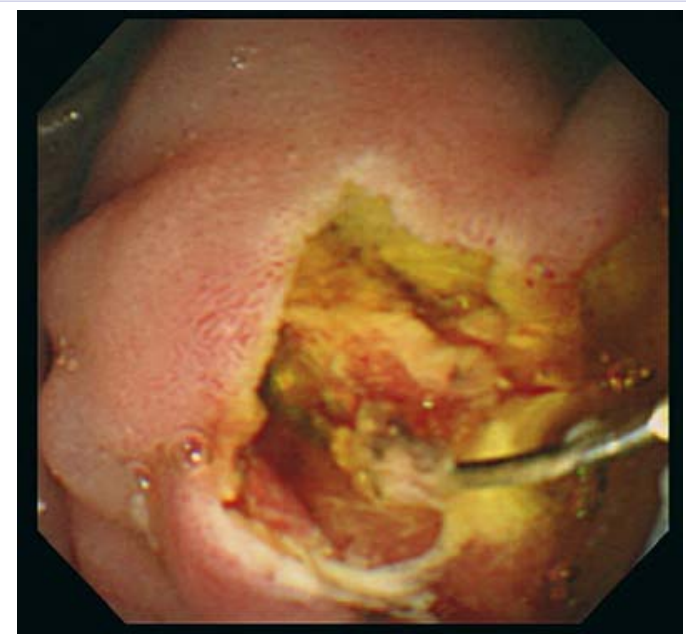

Figure 4: Duodenoscopy revealing the sphincterotomized papilla of Vater. A large, yellowish stone is seen through the incised orifice.

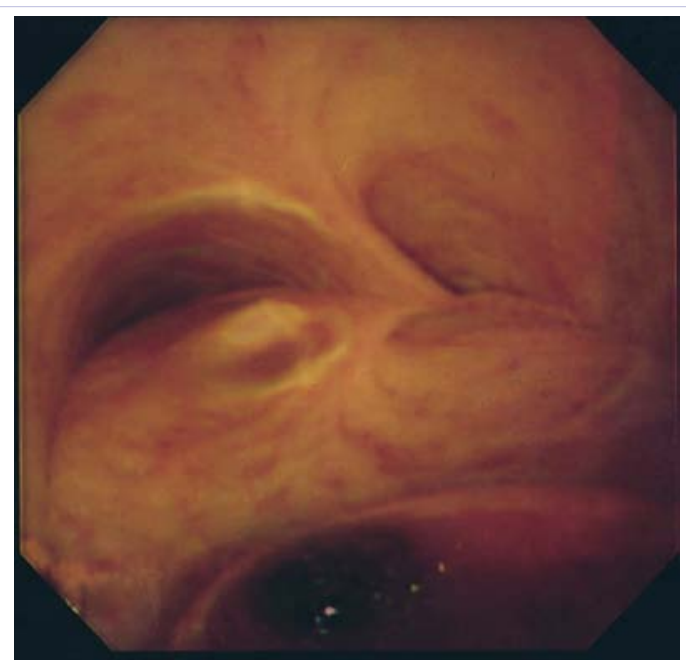

Figure 5a: Peroral cholangioscopy showing the confluent point of the common hepatic duct on the left and the rudimentary cystic duct on the right.

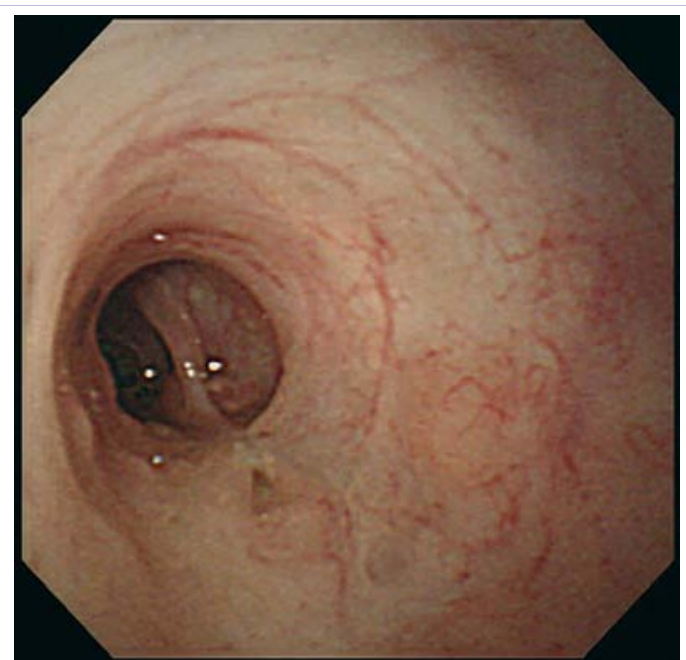

Figure 5b: The inside of the crescent-folded, hypogenetic cystic duct.

Since a case of CAGB was reported to be accompanied with choledochal cyst [19], the blind duct might be a type II choledochal cyst. Peroral cholangioscopy, however, clearly showed it, absolutely different from the dysmorphic cyst of Pierro et al. [20], to be the crescent-folded cul-de-sac originating from and running cephalad parallel with the mid extrahepatic bile duct, definitely revealing it to be the hypogenetic cystic duct, the vestige of the abortive primordium which should have developed into the full-fledged cystic duct and gallbladder. Thus it thoroughly confirmed the preoperative diagnosis and ruled out the possibility of choledochal cyst. The findings obtained by these latter two peroral, diagnostic modalities fulfill the above criteria without resorting to exploration through the non-natural orifices.

A case of CAGB with the absence of the cystic duct was reported to have been diagnosed by ERCP, in which the symptoms 


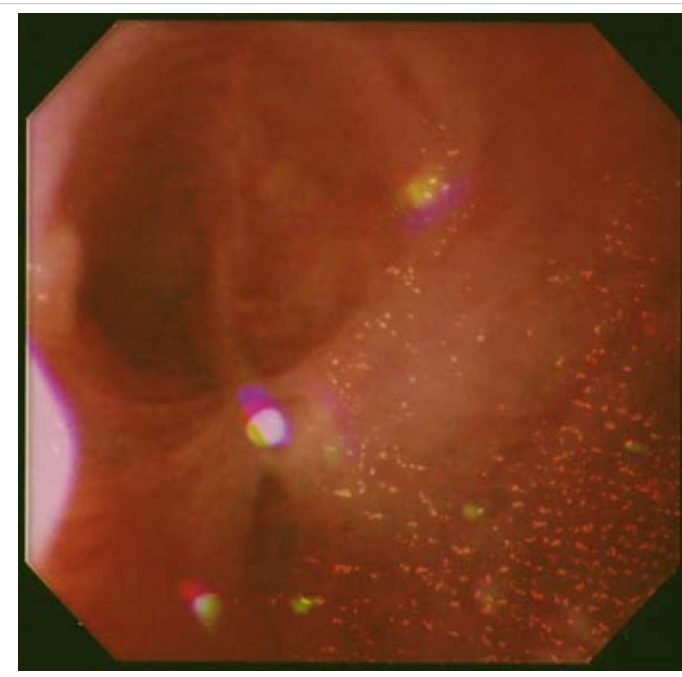

Figure 5c: The blind end of the hypogenetic cystic duct.

were non-specific and no complications were detected and, therefore, no treatment was necessary [21]. There was also a case of CAGB with the hypogenetic cystic duct complicated with choledocholithiasis, in which the final diagnosis was made by laparoscopy combined US after endoscopic choledocholithotomy in the course of attempted laparoscopic cholecystectomy under the diagnosis of severe atrophy of the gallbladder with the cystic duct obstruction [6]. No cases of CAGB, however, have been described, so far, which were definitely diagnosed by ERC and peroral cholangioscopy and successfully treated by non-surgical means under the correct preoperative diagnosis.

\section{Conclusion}

This is the fifth case of CAGB with the hypogenetic cystic duct and the first in the world, which was definitely diagnosed by peroral findings. This report emphasizes the importance of awareness of CAGB and highlights the crucial role of peroral cholangioscopy in diagnosing the anomaly and consequently obviating unnecessary, potentially dangerous exploration through the non-natural orifices in quest of the phantom gallbladder.

\section{References}

1. Monroe SE. Congenital absence of the gallbladder: A statistical study. J Int Coll Surg. 1959; 32: 369-373.

2. Frey C, Bizer L, Ernst C. Agenesis of the gallbladder. Amer J Surg. 1967; 114(6): 917-926. doi: 10. 1016/0002-9610(67)90418-7.

3. Raju SG. Agenesis of the gallbladder in two adults. Brief review of the literature. Am J Gastroenterol. 1972; 57(1): 55-62.

4. Bennion RS, Thompson JE , Tompkins RK. Agenesis of the gallbladder without extrahepatic biliary atresia. Arch Surg. 1988;123(10): 12571260. doi: 10.1001/archsurg.1988.01400340083014.
5. Peloponissios N, Gillet M, Cavin R, Halcic N. Agenesis of the gallbladder: a dangerously misdiagnosed malformation. World J Gastroenterol. 2005; 11(39): 6228-6231.

6. Kwon AH, Yanagimoto H, Matsui Y, Imamura A. Agenesis of the gallbladder with hypoplastic cystic duct diagnosed at laparoscopy. Surg Laparosc Endosc Percutan Tech. 2006; 16(4): 251-254. doi: 10.1097/00129689-200608000-00012.

7. Ishida M, Egawa S, Takahashi $Y$, Kohari M, Ohwada $Y$, Unno M. Gallbladder agenesis with a stone in the cystic duct bud. J Hepatobiliary Pancreat Surg. 2008; 15(2): 220-223. doi: 10.1007/s00534-0071236-3.

8. Bani-Hani KE. Agenesis of the gallbladder: Difficulties in management. J Gastroenterol Hepatol. 2005; 20(5): 671-675. doi: 10.1111/j.14001746.2005.03740.x.

9. Cho CH, Suh KW, Min JS, Kim CK. Congenital absence of gallbladder. Yonsei Med J. 1992; 33(4): 364-367.

10. Azmat N, Francis KR, Mandava N, Pizzi WF. Agenesis of the gallbladder revisited laparoscopically. Am J Gastroenterol. 1993; 88(8):12691270.

11. Aristotle. Peri zoion morion. 667b. Greek.

12. Blechschmidt CM. Agenesis of the gallbladder-Borderline-case of normality? Anat Anz. 1982; 151(3): 281-285.

13. Eheman RL, Morrish HF. Retrorenal gallbladder: A case report. J Can Assoc Radiol. 1983; 34(4): 321-322.

14. Youngwirth LD, Peters JC, Perry MC. The suprahepatic gallbladder. Radiology. 1983; 149(1): 57-58. doi: 10.1148/ radiology.149.1.6611951.

15. Nelson PA, Schmitz RL, Perutsea S. Anomalous position of the gallbladder within the falciform ligament. AMA Arch Surg. 1953; 66(5):679-81.

16. Wysong CB, Gorton RJ. Intrahepatic gallbladder. South Med J. 1980; 73(6): 825-826.

17. Bullard RW. Subcutaneous or extraperitoneal gallbladder. JAMA. 1945; 129(14): 949. doi: 10.1001/jama.1945.92860480005008.

18. Afifi ES, Atef H, Wael B. Agenesis of the gallbladder with primary choledochal stones. Med Princ Pract. 2006; 15: 379-381. doi: 10.1159/000094273.

19. Tabibian JH, Tabibian N, Aguet JC. Choldechal cyst complications presenting as duodenal obstruction in an 82-year-old patient with gallbladder agenesis. Dig Dis Sci. 2009; 54(1): 184-187. doi: 10.1007/ s10620-008-0317-8.

20. Pierro A, Martucci M, Maselli G, Farchione A. Agenesis of the gallbladder with the presence of a small dysmorphic cyst: role of magnetic resonance cholangiopancreatography. J Clin Imaging Sci. 2012; 2: 17. doi:10.4103/2156-7514.95431.

21. O'Sullivan J, O'Brien PA, MacFeely L, Whelton MJ. Congenital absence of the gallbladder and cystic duct: Nonoperative diagnosis. Am J Gastroenterol. 1987; 82(11): 1190-1192. 